\title{
Microwave Conductivity in the Pure and Iodine-Doped Organic Conductor $\alpha$-(BEDT-TTF) $\mathbf{I}_{3}$
}

\author{
W. Kremer, H. W. Helberg \\ Drittes Physikalisches Institut, Universitāt Göttingen, Bürgerstraße 42-44, D-3400 Göttingen \\ E. Gogu, D. Schweitzer \\ Max-Planck-Institut für medizinische Forschung, Abteilung für Molekulare Physik, Heidelberg
}

\author{
H. J. Keller
}

Anorganisch-Chemisches Institut, Universität Heidelberg

\begin{abstract}
Complex Compounds / Electrical Properties / Iodine Doping / Microwave Conductivity / Organic Conductor / Superconductivity
\end{abstract}

The microwave conductivity in dependence of temperature was measured on pure and iodine doped crystals of $\alpha$-(BEDT-TTF) $I_{3}$ along the stack direction [100] and perpendicular to it [010]. The exposure times to iodine were varied in many steps between 2 min and 360 min. The contactless microwave technique enables to keep the same crystal in each case throughout all measurements. - The results show that the microwave conductivity vs. temperature is not influenced distinctly by iodine doping. Only at the onset of the plateau range immediately below the metal-insolator transition at $135 \mathrm{~K}$ a small enhancement of the conductivity is found for exposure times larger than $60 \mathrm{~min}$. - In no case a transition to a superconducting state is observed. Therefore recently reported superconductivity may be due to surface effects.

\section{Introduction}

BEDT-TTF $^{1)}$ salts are of considerable interest since strong intermolecular contacts lead often to two dimensional metallic behaviour down to low temperatures and in some cases even to superconductivity at ambient pressure. The $\alpha$-phase of (BEDT-TTF) ${ }_{2} \mathbf{I}_{3}$ undergoes a metal-insolator (MI) transition at $135 \mathrm{~K}$ at ambient pressure [1]. The conductivity shows a metallic behaviour above the MI transition temperature $\left(T_{\mathrm{MI}}\right)$ and falls down at $T_{\mathrm{MI}}$ by about five orders of magnitude [1]. Recently reported iodine doping of $\alpha$-(BEDT-TTF $)_{2} \mathrm{I}_{3}$ causes a transition to superconductivity in some cases [2]. Cooling down the conductivity goes through a minimum (more than one order of magnitude below room temperature conductivity) immediately below

1) BEDT-TTF or "ET" = Bis(ethylenedithio)tetrathiafulvalene.
$T_{\mathrm{MI}}$ and than rises again, followed by a transition to superconductivity around $3.2 \mathrm{~K}$ at ambient pressure. Reaxamination of these measurements confirms a metallic behaviour below $T_{\mathrm{MI}}$ only in some cases (Fig. 1). But in no case a transition to superconductivity was found. Our own measurements as well as other published measurements [3] of the ac magnetic susceptibility show no indication of superconductivity. The questions are whether the metallic and superconducting states are due to bulk effects or to surface effects and what is the dependence on the duration of exposure to iodine.

Microwave measurements are an useful tool to prove this behaviour. They neglect surface effects and return the properties of the bulk material. Furthermore, they work without contacts. Thus it is possible to measure the same sample repeatedly after each doping procedure [4]. 


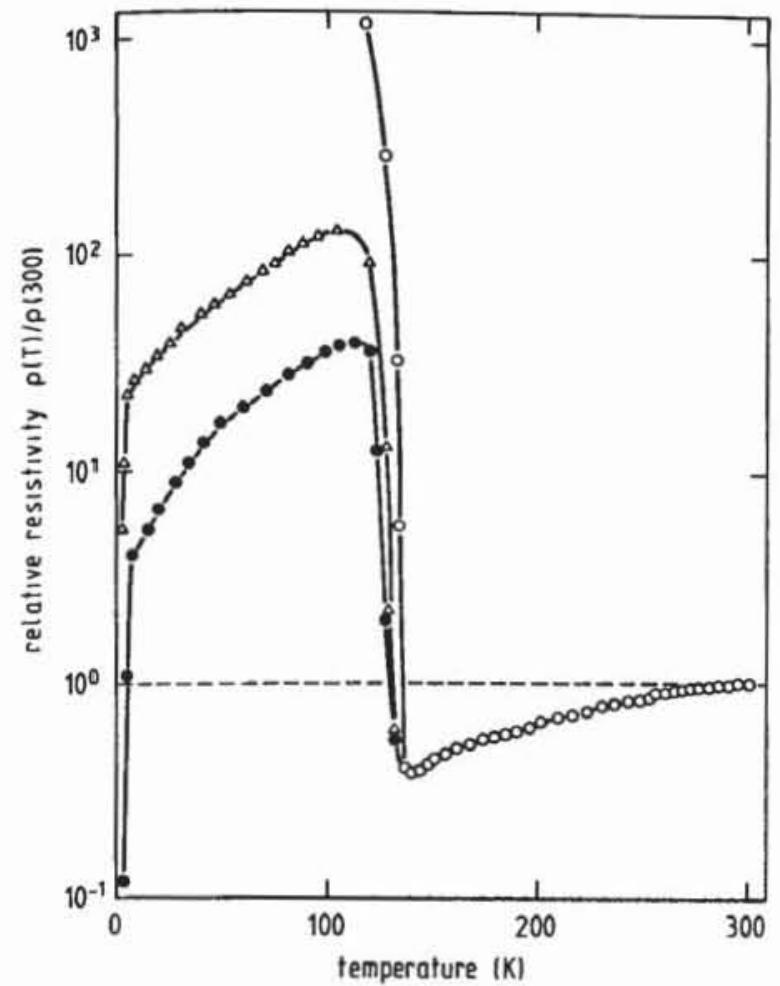

Fig. 1

dc measured relative resistivity in $\alpha$-(ET) $I_{3}$ versus temperature

O undoped (from [1])

$\Delta$ iodine doped without superconductivity (present work)

- iodine doped with superconductivity (from [2])

\section{Experimental}

The $\alpha$-phase of (BEDT-TTF) ${ }_{2} I_{3}$ crystallizes in platelike crystals with plane (001). Measurements of the conductivity were performed using usual four point techniques.

For the microwave measurements small samples approximately square sized were used (length about 1 to $2 \mathrm{~mm}$, thickness about 0.05 to $0.1 \mathrm{~mm}$ ). The crystal orientation of the samples was done with the aid of a polarizing microscope utilizing the maximum absorption of light polarized along the stack direction [100] in the red range of the visible spectrum. The samples were exposed to saturated iodine vapour for different durations. $24 \mathrm{~h}$ and $48 \mathrm{~h}$ after each doping the microwave conductivity was measured along the [010] direction and along the stack direction [100], respectively. [010] stands nearly perpendiculary on [100].

The samples were inserted into a cylindrical cavity working in the $\mathrm{TE}_{111}$-mode at $10 \mathrm{GHz}$. The complex permittivity and thus the microwave conductivity are determined from the frequency shift and the quality change by the usual cavity perturbation technique. The computer controlled measurements were performed during the cooling down process.

\section{Results and Discussion}

In Fig. 1 the dc resistivity is plotted versus temperature for one case in which a sharp decrease of the resistivity below $T_{\mathrm{M} 1}$ was found. The dropping looks similar to that reported by Yagubskii et al. [2]. But in our case there is no transition to superconductivity.

Figs. 2 and 3 contain the microwave conductivity versus temperature for the probe no. 23 in pristine state measured along the stack direction [100] and along the direction [010]. The conductivity has metallic character down to $T_{\mathrm{MI}}$

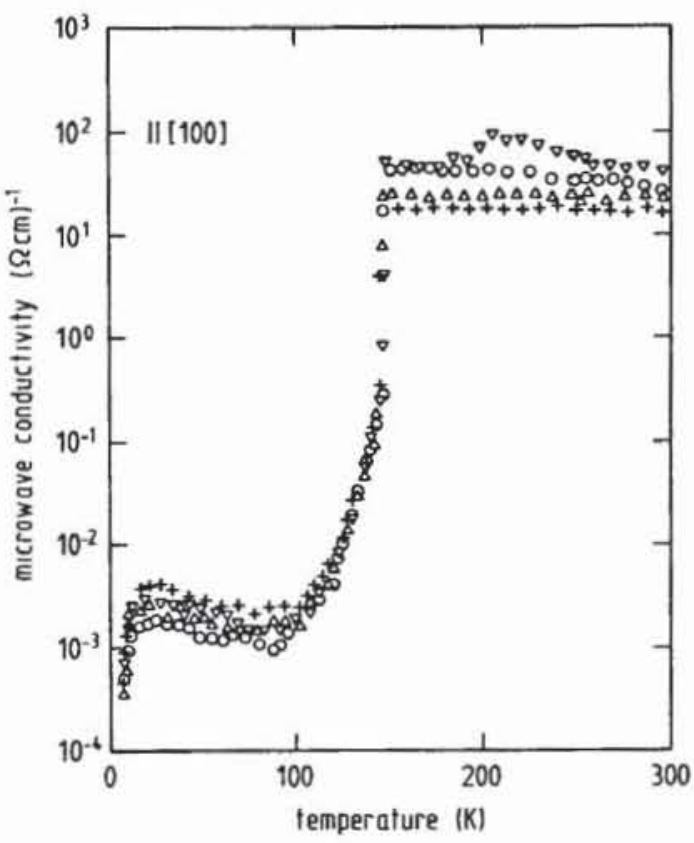

Fig. 2

Microwave conductivity of $\alpha$-(ET) $I_{3}$ for increasing iodine doping versus temperature along stack direction $[100]$ at $10.3 \mathrm{GHz}$ (sample no. 23). Duration of exposure to iodine: $+0 \mathrm{~min}, \triangle 2 \mathrm{~min}, 04 \mathrm{~min}, \nabla 6 \mathrm{~min}$

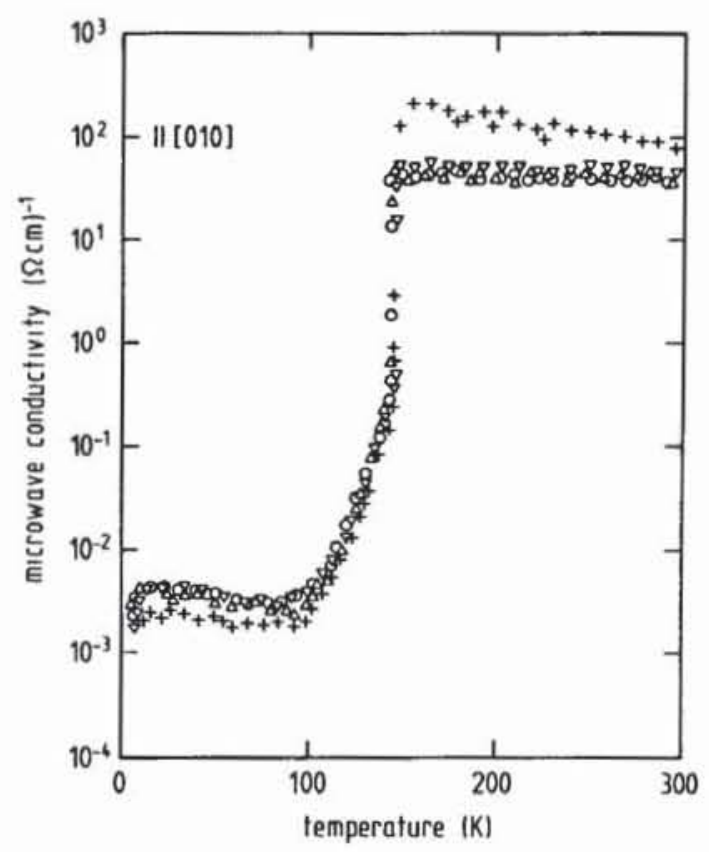

Fig. 3

Microwave conductivity of $\alpha-(\mathrm{ET})_{2} \mathrm{I}_{3}$ for increasing iodine doping versus temperature along direction [010] at $10.3 \mathrm{GHz}$ (sample no. 23). Duration of exposure to iodine: $+0 \mathrm{~min}, \wedge 2 \mathrm{~min} .04 \mathrm{~min}, \nabla 6 \mathrm{~min}$

and is some-what higher in the [010] direction than in stack direction in this range. At $T_{\mathrm{MI}}$ the conductivity drops by more than 3 orders of magnitude and change then to a plateaulike range between 120 and $20 \mathrm{~K}$. Below $20 \mathrm{~K}$ the conductivity falls down again.

This behaviour is consistent with earlier published data $[1,4]$. In contrast to microwave measurements the dc con- 
ductivity continues to decrease approximately with an activated character $(\approx 0.08 \mathrm{eV})$ after a rapid drop at $T_{\mathrm{MI}}[1]$. The origin of the plateau behaviour found by microwaves is not yet clearly understood. The frequency dependence [4] of the conductivity below $T_{\mathrm{MI}}$ might be accounted for by charge density waves, which can be pinned by impurities of disorder. The crystal structures above and below $T_{\mathrm{MI}}$ were determined [5-6] to examine the nature of the MI transition. Neither a superstructure nor a change of the space group symmetry due to the MI transition was found. There is only a small change in the triiodide network and in the degree of dimerization of one of the donor stacks.

Subsequently the crystal sample no. 23 was successively step-by-step exposed to iodine. The duration of exposure started with 2 min and was increased summing up to the following effective durations of exposure: $2,4,6,7,9,11,13$, $15,17,20,25,30,40,50,60,90,120,180$, and $360 \mathrm{~min}$. After each doping procedure the microwave conductivity was measured in both directions.

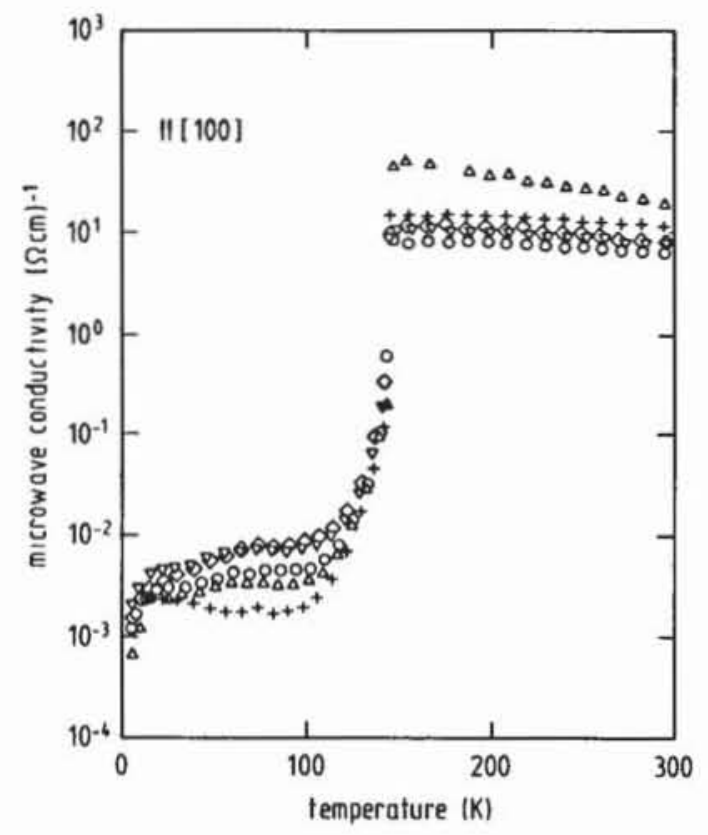

Fig. 4

Microwave conductivity of $\alpha$-(ET) $I_{3}$ for increasing iodine doping versus temperature along stack direction [100] at $10.3 \mathrm{GHz}$ (sample no. 23). Duration of exposure to iodine:

$+60 \mathrm{~min}, \triangle 90 \mathrm{~min}, \bigcirc 120 \mathrm{~min}, \nabla 180 \mathrm{~min}, \diamond 360 \mathrm{~min}$

Figs. 2 and 3 show the microwave conductivity for doping durations up to $6 \mathrm{~min}$ and Figs. 4 and 5 for durations from 60 to $360 \mathrm{~min}$, respectively. Fig. 6 summarizes the measured microwave conductivities at $300 \mathrm{~K}$ and at $60 \mathrm{~K}$ of the whole series of doping durations.

There is no significant dependence on doping with iodine. Only for exposure durations larger than $60 \mathrm{~min}$ a small enhancement of the conductivity below $T_{\mathrm{MI}}$ at the onset of the plateau is observed somewhat more pronounced for the [010] direction.

The reason for the somewhat higher conductivity in the [010] direction with respect to the stack direction [100] particularly in the metallic range above $T_{\mathrm{MI}}$ is because of a difference of the interactions between the BEDT-TTF molecules in these directions. There are four intrastack $\mathbf{S} \cdots \mathrm{S}$

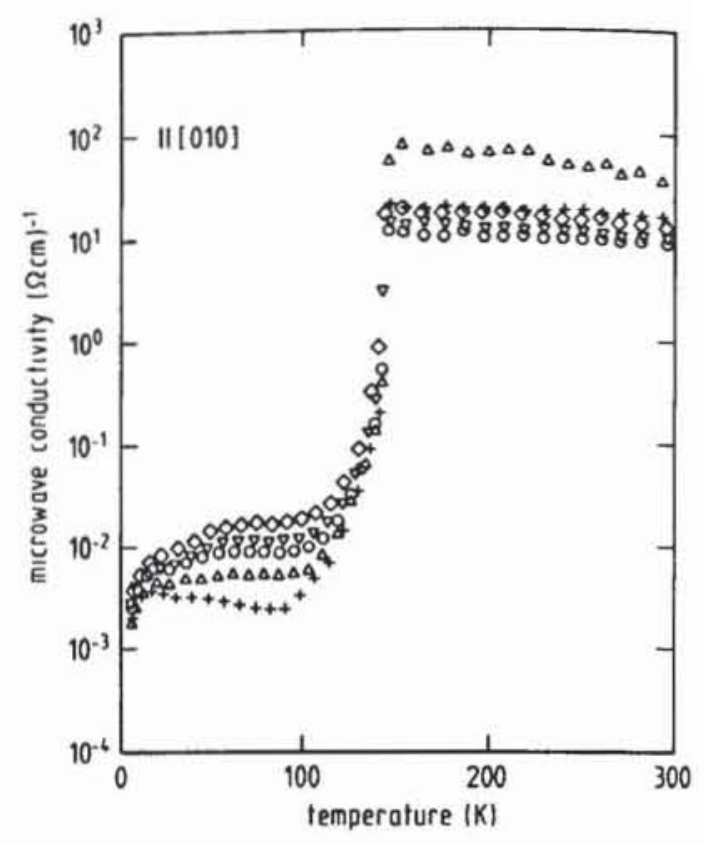

Fig. 5

Microwave conductivity of $\alpha-(\mathrm{ET})_{2} l_{3}$ for increasing iodine doping versus temperature along direction $[010]$ at $10.3 \mathrm{GHz}$ (sample no. 23). Duration of exposure to iodine:

$+60 \mathrm{~min}, \triangle 90 \mathrm{~min}, \bigcirc 120 \mathrm{~min}, \nabla 180 \mathrm{~min}, \diamond 360 \mathrm{~min}$

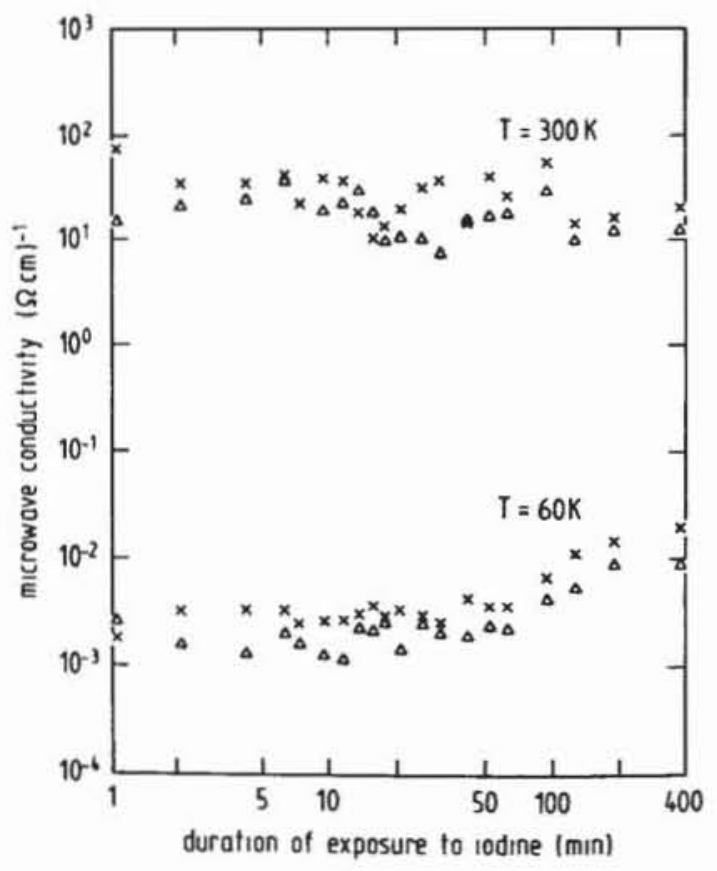

Fig. 6

Microwave conductivities $(10.3 \mathrm{GHz})$ of sample no. 23 of $\alpha-(\mathrm{ET})_{2} \mathrm{I}_{3}$ at $60 \mathrm{~K}$ and $300 \mathrm{~K}$ for both crystal directions collected from all measurements at the different durations of exposure to iodine. Values for the pristine state inserted at $1 \mathrm{~min}$ $\triangle$ parallel [100], $\times$ parallel [010]

contacts, but seven interstack $\mathrm{S} \cdots \mathrm{S}$ contacts between adjacent BEDT-TTF molecules. Furthermore, the interstack S $\cdots$ S contact distances are shorter than the intrastack contact distances. The interstack $\mathbf{S} \cdots \mathrm{S}$ contact directions are arranged in a symmetrical $60^{\circ}$ network in space related to the parallel long in-plane molecular axes of the BEDT-TTF molecules [7]. Thus two ribbon-like side-by-side connections are formed extended along the directions inclined by 
$11^{\circ}$ and $49^{\circ}$, respectively, to $c^{*}$ in the plane $\left(b^{\prime}, c^{*}\right)$ perpendicular to the stack direction [100] resulting in a pronounced interaction perpendicular to the stack. This is supported by calculations of the interaction energies [6].

The two unique triiodide chains form layers which separate the layers of the two-dimensional BEDT-TTF network. Examination of the space between the triiodide chains show that the insertion of excess iodine may be possible. It is assumed that for long exposure durations ( $\geq 60 \mathrm{~min}$ ) with iodine the excess iodine diffuses increasingly deeper into the triiodine layers leading to specific interactions with the adjacent BEDT-TTF network. The small enhancement of the microwave conductivity in the plateau range shown in Figs. 4 and 5 after long doping times may be the result of such diffusion processes into the bulk material.

Nevertheless, in no case neither a transition to the superconducting state nor a sharp metal-like increase of the conductivity below $T_{\mathrm{M} 1}$ (like Fig. 1) in the whole observed doping range up to $3 \mathrm{~h}$ was found. Thus the observed superconductivity or the high conductivity, respectively, are probably due to the effects on the surface of the samples.
We thank the Stiftung Volkswagenwerk for supporting this work.

\section{References}

[1] K. Bender, K. Dietz, H. Endres, H. W. Helberg, I. Hennig, H. J. Keller, H. W. Schäfer, and D. Schweitzer, Mol. Cryst. Liq. Cryst. 107, 45 (1984).

[2] E. B. Yagubskii, J. F. Shchegolev, V. N. Laukhin, P. A. Konovich, M. B. Kratsovnik, A. V. Zvarykina, and L. J. Buravov, Pis'ma Zh. Eksp. Teor. Fiz 39, 12 (1984).

[3] H. Veith, C. P. Heidmann, C. P. Gross, T. Leit, A. Andres, and D. Schweitzer, Solid State Commun. 56, 1015 (1985).

[4] M. Przybylski, H. W. Helberg, D. Schweitzer, and H. J. Keller, Synth. Metals 19, 191 (1987).

[5] H. Endres, H. J. Keller, R. Swietlik, D. Schweitzer, K. Angermund, and C. Krüger, Z. Naturforsch. $41 a, 1319$ (1986).

[6] T. J. Emge, P. C. W. Leung, M. A. Beno, H. H. Wang, and J. M. Williams, Mol. Cryst. Liq. Cryst. 138, 393 (1986).

[7] H. W. Helberg, Synth. Metals 19, 251 (1987).

Presented at the Discussion Meeting of the Deutsche Bunsen-Gesellschaft für Physikalische Chemie "Physics and Chemistry of Unconventional Organic Materials", Wiesbaden-Naurod, April 29th to May 1st, 1987 